\title{
The meandering instability of a viscous thread
}

\author{
Stephen W. Morris ${ }^{1}$, Jonathan H. P. Dawes ${ }^{2}$, Neil M. Ribe ${ }^{3}$, and John R. Lister ${ }^{2}$ \\ ${ }^{1}$ Department of Physics, University of Toronto, 60 St. George St., Toronto, Ontario, Canada M5S $1 A 7$ \\ ${ }^{2}$ DAMTP, Centre for Mathematical Sciences, University of Cambridge, Wilberforce Road, Cambridge CB3 0 WA \\ 3 Dynamique des Fluides Géologiques, IPGP et Université de Paris-7, \\ CNRS, Tour 14, 2, place Jussieu, 75005 Paris, France
}

(Dated: October 29, 2018)

\begin{abstract}
A viscous thread falling from a nozzle onto a surface exhibits the famous rope-coiling effect, in which the thread buckles to form loops. If the surface is replaced by a belt moving with speed $U$, the rotational symmetry of the buckling instability is broken and a wealth of interesting states are observed [See S. Chiu-Webster and J. R. Lister, J. Fluid Mech., 569, 89 (2006)]. We experimentally studied this "fluid mechanical sewing machine" in a new, more precise apparatus. As $U$ is reduced, the steady catenary thread bifurcates into a meandering state in which the thread displacements are only transverse to the motion of the belt. We measured the amplitude and frequency $\omega$ of the meandering close to the bifurcation. For smaller $U$, single-frequency meandering bifurcates to a two-frequency "figure eight" state, which contains a significant $2 \omega$ component and parallel as well as transverse displacements. This eventually reverts to single-frequency coiling at still smaller $U$. More complex, highly hysteretic states with additional frequencies are observed for larger nozzle heights. We propose to understand this zoology in terms of the generic amplitude equations appropriate for resonant interactions between two oscillatory modes with frequencies $\omega$ and $2 \omega$. The form of the amplitude equations captures both the axisymmetry of the $U=0$ coiling state and the symmetrybreaking effects induced by the moving belt.
\end{abstract}

PACS numbers: 82.40.Bj, 47.20.Gv, 47.20.Ky

The fascinating instabilities of very viscous fluids are familiar to anyone who has poured syrup onto a pancake [1. The thread of syrup elongates as it falls, and exhibits a buckling instability as nears the surface [2]. The buckling is the result of a competition between bending and axial compression, and causes the thread to loop itself into coils. This "liquid rope coiling effect" 3. has a long history comprising almost a half century of experiments [2, 3, 4, 5, 6, 7, scaling analyses [7, 8, 9, analytic theory and numerical simulation [7, 10, 11, 12, 13, 14. It has been observed that the coils tend to pile up on the surface, forming a tall column [3, 4, 5] and that the frequency of coiling exhibits a complex, multivalued dependence on the fall height [5, 7. Here we consider a variation on this classic experiment, the case when the liquid thread falls onto a moving surface, a situation known as the "fluid-mechanical sewing machine" [15] owing to the variety of patterns deposited on the surface.

The moving surface breaks one of the basic symmetries of the "pure" rope coiling problem, and has the effect of unfolding the coiling instability into a rich panoply of distinct bifurcations. This arrangement is also very convenient experimentally; the moving surface is provided by a belt which is cleared of fluid before it returns, eliminating the pile-up of viscous fluid at the point of contact.

The main experimental control parameters are the height $H$ of the nozzle from which the thread descends, and the speed of the moving surface $U$. Less important parameters, which we hold fixed in this work, are the volumetric flow rate of the liquid $Q$ and the diameter of the nozzle $d$. The Newtonian fluid is characterized by its density $\rho$, kinematic viscosity $\nu$ and surface tension $\sigma$. Non-Newtonian effects are negligible.
We present an experimental study of the "stitch" patterns made by the thread as it is laid down on the belt, as a function of $H$ and $U$. We first discuss a survey of the states in the $H-U$ plane, with denser coverage than was possible in Ref. 15. Then we focus on the simplest bifurcation, that from the straight catenary state to meandering. We show that this state is well described as a forward Hopf bifurcation and compare the onset belt speed and Hopf frequency to recent linear stability theory [16. We examine the nonlinear saturation of the meandering amplitude and qualitatively explain it with a simple kinematic model of an inextensible thread. We then essay the task of understanding the more complex "figure 8" and other patterns that appear as the belt speed is lowered. We propose a general amplitude equation approach, based on the idea that the motion of the belt can be treated as a perturbation to the pure rope coiling problem which has $O(2)$ symmetry. We propose a set of coupled amplitude equations which minimally break $O(2)$ symmetry, and mix amplitudes for rotating wave modes with frequencies $\omega$ and $2 \omega$. We conjecture that all the complicated stitch patterns can be captured by this approach, which could be generalized to include more modes as they become excited near the multifrequency regime. These modes can be directly measured by visualizing longitudinal and transverse motions of the thread by viewing from the side.

This paper is organized in the following way. In section II. we discuss the fluid properties and the experimental apparatus. In section II, we make comparisons between our parameters and the corresponding zero belt speed, pure coiling problem, before turning to the state diagram in section II A and the meandering threshold in sections 
IIB and IIC In section IID we discuss experimental observations of the states beyond meandering. Section III presents our general amplitude formalism which provides a consistent framework for interpreting the experimental results. We have placed a detailed discussion of the derivation of the amplitude equations in an Appendix. Section IV is a brief conclusion.

\section{EXPERIMENT}

Our experimental apparatus was a redesigned version of the one described in Ref. 15. It is shown schematically in Fig. 1. The main differences were improved control and measurement of the belt speed, and the use of very stable silicone oil as the working fluid.

Silicone oil offers several advantage over the Lyle's Golden Syrup used in previous experiments [15. Although its viscosity cannot be varied by adding water as described in Ref. 15, silicone oil is not susceptible to evaporation and its viscosity varies much less with temperature than that of sugar syrup. This makes it possible to do highly reproducible experiments over a long period of time. It also has about one third the surface tension of syrup, which reduces the thread-thread and thread-belt interaction after the fluid is laid down on the belt.

We used Dow Corning 200 (c) fluid, which has a nominal kinematic viscosity of 30,000 cSt. To fully characterize the fluid, we measured its viscosity and the temperature coefficient of its viscosity using a TA AR1000 rheometer. The same instrument was used to confirm that the fluid is Newtonian to an excellent approximation. We also measured the fluid density and its temperature coefficient using an Anton-Parr DMA 5000 densitometer. The results of these measurements are shown in Table I

The temperature of the experiment was recorded during each run, but not otherwise controlled. The only significant variation in the fluid properties was therefore due to the temperature, and not to the degradation of the fluid. Averaged over all experiments, the mean temperature was $22.2 \pm 0.8^{\circ} \mathrm{C}$. Accounting for this systematic error, the fluid had a density of $\rho=996 \pm 8 \mathrm{~kg} / \mathrm{m}^{3}$ and a kinematic viscosity of $\nu=(2.77 \pm 0.02) \times 10^{-2} \mathrm{~m}^{2} / \mathrm{s}$, or $27,700 \pm 200$ cSt.

We used an internally reinforced, toothed timing belt of width $16 \mathrm{~mm}$. The belt was looped over toothed pulleys and driven with a speed-controlled DC motor. The speed of the belt $U$ was measured using an averaging storage oscilloscope to record the intensity of a laser beam that was interrupted by the teeth of the belt. The frequency of the passage of the teeth, measured by an internal function of the oscilloscope, could be used to find $U$ to within a few $\%$. The reinforced timing belt resisted stretching or slipping, even under considerable pressure from the plastic scraper used to remove the silicone oil. After scraping, the belt had only a very thin wetting layer of oil left on it. The excess oil dripped off the scraper into a beaker, and could be recirculated.

\begin{tabular}{|l|c|}
\hline Density $\rho$ & $996 \pm 8 \mathrm{~kg} / \mathrm{m}^{3}$ \\
Temperature coefficient of $\rho$ & $-0.0885 \% /{ }^{\circ} \mathrm{C}$ \\
Molecular viscosity $\eta$ & $27.63 \pm 0.05 \mathrm{~Pa} \mathrm{~s}$ \\
Temperature coefficient of $\eta$ & $-1.6 \% /{ }^{\circ} \mathrm{C}$ \\
Kinematic viscosity $\nu=\eta / \rho$ & $(2.77 \pm 0.02) \times 10^{-2} \mathrm{~m}^{2} / \mathrm{s}$ \\
\hline Maxwell time $\tau_{M}$ & $<1.8 \mathrm{~ms}$ \\
Young's modulus $G$ & $<14 \mathrm{kPa}$ \\
\hline Surface tension $\sigma$ & $21.5 \mathrm{mN} / \mathrm{m}$ \\
\hline
\end{tabular}

TABLE I: The measured properties of the silicone oil at the mean experimental temperature of $22.2 \pm 0.8^{\circ} \mathrm{C}$. The size of the measured Maxwell time and Young's modulus show that viscoelastic effects are negligible. The surface tension is taken from the manufacturer's data sheet.

In all the experiments reported here, we used a nozzle with $d=8.00 \pm 0.02 \mathrm{~mm}$ to deliver a fixed, constant volumetric flux of oil $Q$. The oil was supplied by a reservoir and driven by a gravity feed system with a fixed $42 \mathrm{~cm}$ head. The reservoir and the nozzle were attached to a frame mounted on a screw so that they travelled together, maintaining a constant head as the fall height $H$ was varied. $H$ was measured with a scale to $\pm 0.05 \mathrm{~cm}$.

Before and after each run, we weighed the quantity of oil delivered in a certain time interval, typically $2 \mathrm{~min}$, timed with a stopwatch. We found the mass flux $\rho Q=$ $0.0270 \pm 0.0006 \mathrm{~g} / \mathrm{s}$ remained constant within experimental error, even for runs performed many days apart. All the experiments we discuss here used this value of $\rho Q$.

An experimental run consisted of fixing the nozzle height $H$ and varying the belt speed $U$, starting from high values of $U$ for which the thread is dragged horizontally by the belt to form a steady, stretched catenary. Lowering the belt speed to zero, and then increasing it again produces a sequence of bifurcations, and a sequence of "stitch patterns" of the thread on the belt. Care was taken to change $U$ slowly near the onset of the first instability, to meandering, where the state of the thread responded very slowly to small changes in $U$. We recorded the oil pattern on the moving belt from above using a 3 megapixel digital camera. To avoid heating the oil, and to freeze the motion of the thread, the only lighting used was a fast photo flash. Typical photos of the main states of interest are shown in Fig. 22. These and many other states are shown and described in Ref. [15].

\section{RESULTS AND DISCUSSION}

In this section we will tour the zoology of states and bifurcations exhibited by the viscous thread falling on the moving belt. After a general overview, we focus on the simplest transition first, the onset of the meandering state from the stretched catenary as the belt speed is lowered. We compare our results to the existing linear stability theory for this transition 16. Moving into the nonlinear regime, we find that the amplitude of the me- 


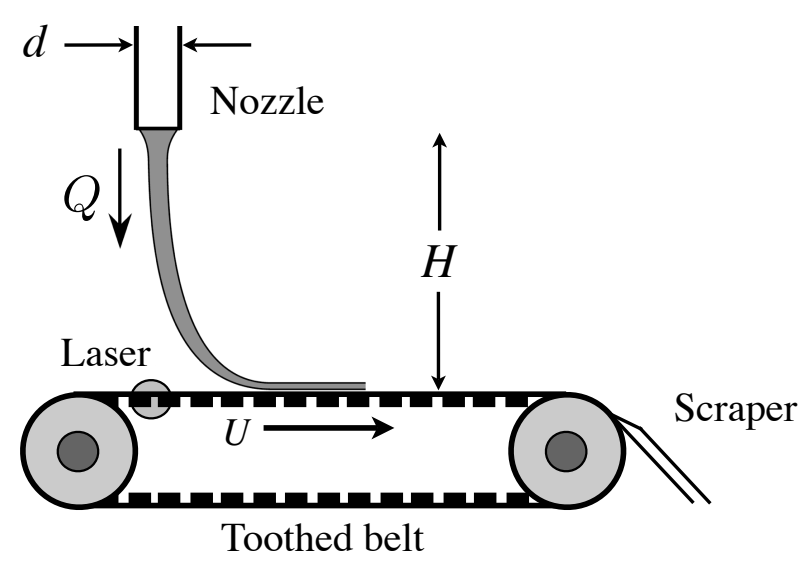

FIG. 1: A schematic of the apparatus. A silicone oil stream with volumetric flow rate $Q$ falls a distance $H$ from a nozzle of diameter $d$ onto a belt moving at speed $U$. The oil is removed by the scraper. The laser, which was interrupted by the teeth of the belt, was used to measure the speed $U$.

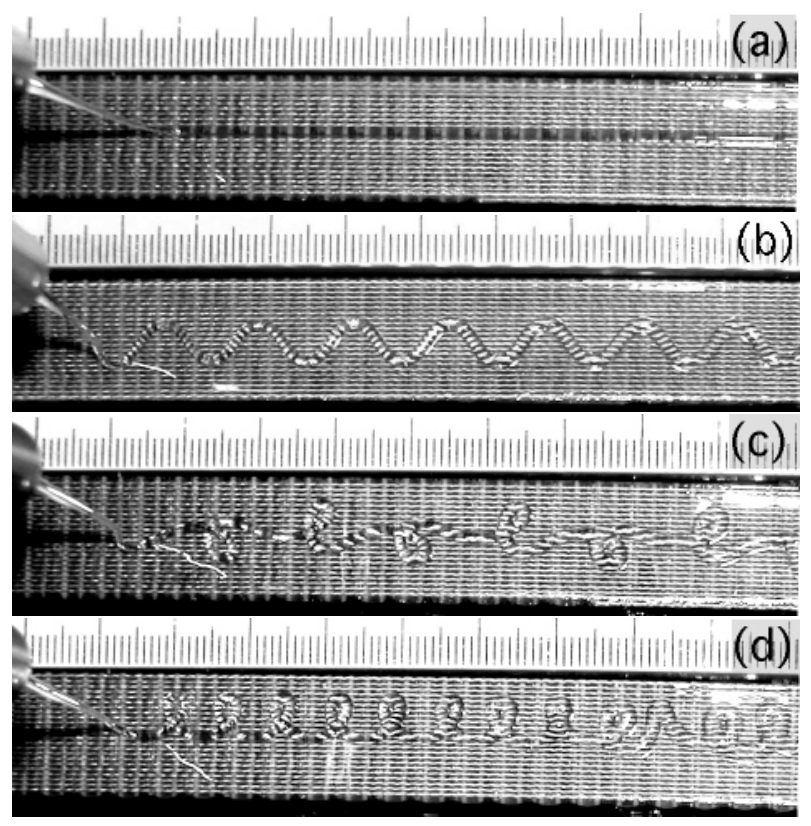

FIG. 2: Photos of some of the states of the thread on the belt. The nozzle is on the left and the belt moves from left to right. The internal reinforcing mesh of the semi-transparent belt is visible, but the surface of the belt is smooth. The scale is in $\mathrm{mm}$. (a) The catenary steady state. (b) The meandering state. (c) The figure eight state. (d) The translated coiling state.

andering is well described as a forward Hopf bifurcation.

We then show that the saturation coefficient of the amplitude can be deduced reasonably well from a simple model of an inextensible thread. Finally, we consider the subsequent bifurcations for small nozzle heights $H$, namely the appearance of the figure eight and translated coiling states. These and the other more intricate states for larger $H$ are probably best understood from the dynamics of their amplitudes. We present a brief outline of a bifurcation theoretic approach to this problem.

At the outset, it is instructive to identify the states of "pure" rope coiling that we would expect to see if the speed of the belt in our experiments were zero. Theory and laboratory experiments show [7, 13, 14, that pure coiling can occur in four different regimes - viscous (V), gravitational $(\mathrm{G})$, inertio-gravitational (IG), and inertial (I) - depending on the relative magnitudes of the different forces acting on the thread. In the V, G, and I regimes, the coiling frequency $\Omega$ is a single-valued function of the fall height $H$. In IG coiling, by contrast, $\Omega(H)$ is multivalued, reflecting the fact that the nearly vertical upper part of the thread acts as a distributed pendulum with multiple resonantly excited eigenmodes.

In a typical experiment where the fluid properties, the flow rate $Q$ and the nozzle diameter $d$ are held constant, the regime that obtains is determined by the fall height $H$. As $H$ increases, the regimes always succeed each other in the order V-G-IG-I. However, while the G and I regimes can be observed for nearly any choice of $Q, d$, and the working fluid, the V and IG regimes are confined to more restricted regions of parameter space. For a given experiment, this space is completely characterized by the three dimensionless groups,

$$
\begin{array}{ll}
\Pi_{1}=\left(\frac{\nu^{5}}{g Q^{3}}\right)^{1 / 5} & {[610 \pm 10]} \\
\Pi_{2}=\left(\frac{\nu Q}{g d^{4}}\right)^{1 / 4} & {[0.370 \pm 0.002]} \\
\Pi_{3}=\frac{\sigma d^{2}}{\rho \nu Q} & {[1.84 \pm 0.06]}
\end{array}
$$

where $g$ is the acceleration due to gravity, $\sigma$ is the surface tension, and the values of each group for our experiments are given in square brackets. For a fluid such as silicone oil with a relatively low surface tension, the value of $\Pi_{3}$ has only a minor effect on the coiling behavior, which is controlled primarily by $\Pi_{1}$ and $\Pi_{2}$. It turns out that our values $\Pi_{1} \approx 610$ and $\Pi_{2} \approx 0.37$ are within the domain where IG coiling is expected to occur for some range of heights (See Ref. [7, Fig. 3.) By contrast, the V regime does not occur, because the characteristic height $(\nu Q / g)^{1 / 4} \approx 3 \mathrm{~mm}$ over which gravitational stretching of the thread becomes important is less than the diameter of the nozzle (as usual with a gravity feed system). A direct numerical calculation using the method of [13] shows that $\mathrm{G}$ coiling is expected in our experiments for $2 \mathrm{~cm}$ $<H<5.7 \mathrm{~cm}$, IG coiling for $5.7 \mathrm{~cm}<H<8.5 \mathrm{~cm}$, and I coiling for $H>8.5 \mathrm{~cm}$. The experiments reported here explore the range $2 \mathrm{~cm}<H<8 \mathrm{~cm}$, with belt speeds up to $U=10 \mathrm{~cm} / \mathrm{s}$. 


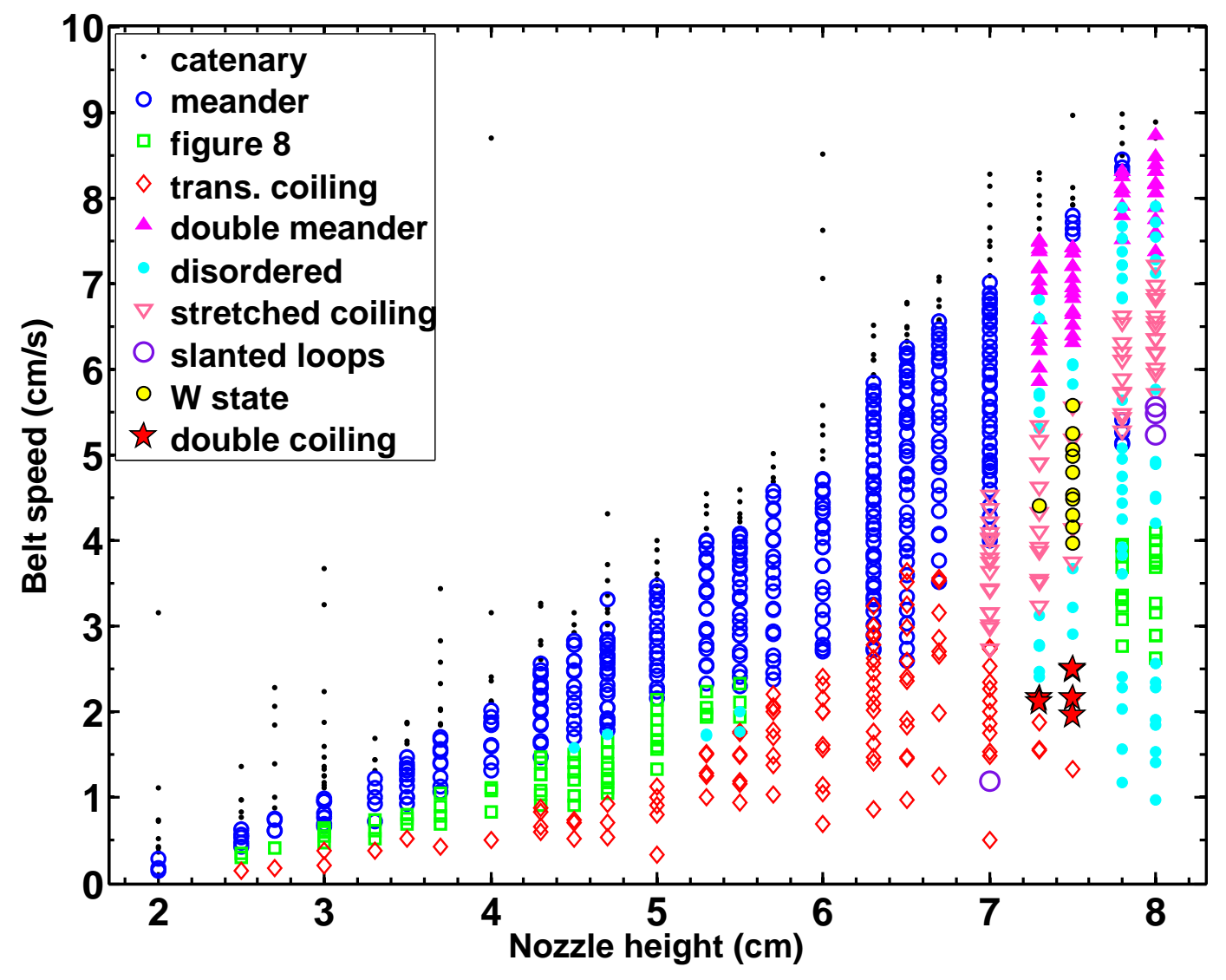

FIG. 3: The state diagram, as a function of the belt speed $U$ and the nozzle height $H$. Some of the simpler states are shown in the previous figure; more complex states are described in Ref. [15].

\section{A. The state diagram}

Fig. 3 shows the states observed in the $U-H$ plane. The diagram was made by scrutinizing over 1100 individual photos. In general, the diagram agrees very well with Fig. 6 of Ref. [15], although certain details differ at larger $H$. At large $U$, only the stretched catenary steady state is seen. As $U$ is reduced at fixed $H$, the first bifurcation is to the meandering state. This transition, which is non-hysteretic for $H \leq 7.0 \mathrm{~cm}$, is discussed in detail below. Above $H=7.0 \mathrm{~cm}$, the meandering state shows clear multifrequency behavior and a small hysteresis at onset. For $H \leq 5.5 \mathrm{~cm}$, as $U$ is reduced, the meandering state switches to a figure eight mode, before making a second transition to translated coiling. All these transitions are very nearly non-hysteretic. It is not clear if the figure eight window extends all the way down to the onset of any instability, just below $H=2.0 \mathrm{~cm}$. For $5.7 \mathrm{~cm} \leq H \leq 7.0 \mathrm{~cm}$, the figure eight state disappears, and there is a small hysteretic region where meandering and coiling co-exist. For $H \geq 7.0 \mathrm{~cm}$, many complex states are observed, with windows of various pat- terns separated by disordered, possibly chaotic regions. In contrast to Ref. [15, almost no region of slanted loops is observed. This may be due to the reduced surface tension of the silicone oil, which reduces the tendency of the thread to stick to itself. Regions of W-states and double coiling are seen, as well as small re-entrant regions of the simpler figure eight and single-frequency meandering modes. The latter was not observed in Ref. 15. We do not observe side-kicks, which may require still larger $H$.

A complete elucidation of the multifrequency states for $H \geq 7.0 \mathrm{~cm}$ must await more precise and better instrumented experiments. For the remainder of this paper, we will focus on the simpler bifurcations between the steady and meandering states, and on the region of the figure eight window for $H \leq 5.5 \mathrm{~cm}$.

\section{B. The threshold of single frequency meandering}

We now consider the bifurcation from the steady stretched state to meandering, which has been analyzed at the linear stability level in Ref. 16. In particular, lin- 
ear theory provides clear predictions for the critical belt speed $U_{c}$ at which meandering begins as $U$ is decreased, and for the critical frequency $\omega_{c}$ of the meandering state which emerges. Quite remarkably, the onset angular frequency $\omega_{c}$ (which, as we will show, is the Hopf frequency that obtains for a zero amplitude of meandering) is predicted to be nearly identical to the frequency expected for pure, nonzero amplitude rope coiling onto a stationary surface for the same $H$ [16.

To accurately locate the onset of meandering, we photographed the state while very slowly decreasing and increasing $U$ through $U_{c}$. We then found the peak-topeak amplitude $2|A|$ and wavelength $\lambda$ of the meandering thread directly from the digital photos. It was typically possible to use data spanning 5 to 10 wavelengths, and the resolution of the images was sufficiently high that the width of the thread was approximately 10 pixels. The smallest amplitudes we could measure were a fraction of the width of the thread, while the largest spanned the belt.

We fit the amplitude data to a phenomenological amplitude model of the Landau form

$$
\tau \dot{A}=\epsilon A-\mu A|A|^{2}+\text { h.o.t., }
$$

with $\epsilon=\left(U_{c}-U\right) / U_{c} . \tau$ is a time scale related to the linear growth rate. We consider the steady state of this equation, so $\tau \dot{A}=0$ and use the fit to determine $U_{c}$ and the saturation coefficient $\mu$. Fig. 4 shows such a fit. The fits were best near the midrange of the heights $H$, declining in quality for small $H$ due to sparsity of data and for larger $H$ due to the growth of the higher order terms. Near $H \approx 5 \mathrm{~cm}$, the amplitude has an essentially perfect square-root dependence $|A|=\sqrt{\epsilon / \mu}$, as shown in Fig. 4. For $H>7.0 \mathrm{~cm}$, fitting was no longer possible, and we simply estimated $U_{c}$ by bracketting the onset of multifrequency meandering.

Fig. 5 shows the critical belt speed $U_{c}$ as a function of the nozzle height $H$, compared to the prediction of the linear stability theory [16. There are no adjustable parameters in the theory. The backward looping portions of the theoretical curve mark the inertial-gravitational regime where multifrequency coiling would exist in the pure rope coiling case. Since our experimental protocol was to fix $H$ and vary $U$, we would expect to observe the upper envelope of this curve. The agreement is very satisfactory.

We have no immediate explanation for the slight systematic discrepancy by which the theory overestimates $U_{c}$ by an amount that increases with $H$. Presumably it is due to some unaccounted for physical effect, such as air drag or surface tension effects at the contact point between the thread and the belt. For large $H$, the thread is very sensitive to air currents and the rapidly moving belt inevitably disturbs the air nearby.

The meander frequency $\omega$ can also be easily obtained from the measurements of the wavelength $\lambda$ of the meander pattern on the belt. The angular frequency is simply $\omega=2 \pi U / \lambda$. We find that $\omega$ decreases linearly with $U$,

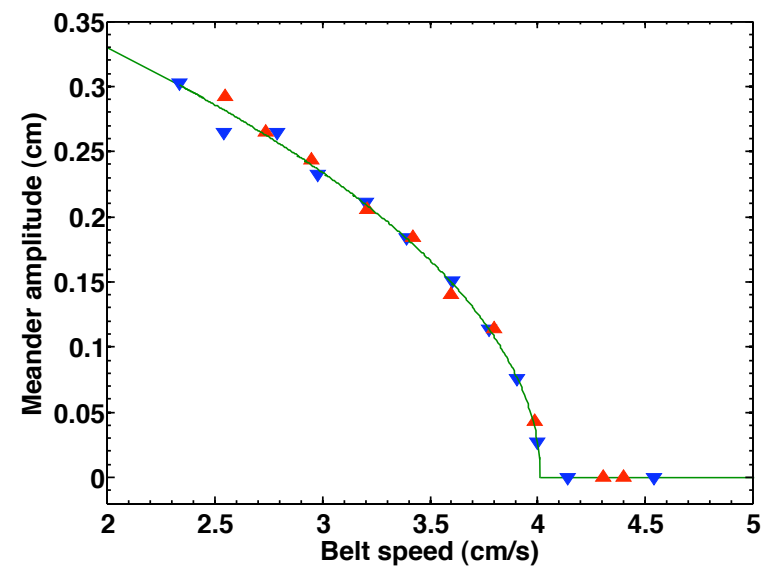

FIG. 4: (Color online) The amplitude $|A|$ of the meandering motion as a function of the belt speed $U$, for $H=5.3 \mathrm{~cm}$. The upward (downward) pointing triangles indicate data taken with increasing (decreasing) $U$. The solid line is a fit to Eqn. 4 with $U_{c}=4.01 \mathrm{~cm} / \mathrm{s}$ and $\mu=4.62 \mathrm{~cm}^{-2}$. No higher order terms were required. The smallest nonzero amplitudes here are about half the width of the thread.

so that it is straightforward to extrapolate to obtain $\omega_{c}$ as shown in Fig. 6. The functional forms of the amplitude and frequency of meandering shown in Figs. 4 and 6 demonstrate that the onset of single frequency meandering is very well described as a forward Hopf bifurcation with Hopf frequency $\omega_{c}$. This onset frequency is also predicted by linear stability theory [16]; Fig. 7] shows the Hopf frequency as a function of $H$. Here the agreement with the theory is excellent, and again there are no adjustable parameters.

It is interesting that the frequency measurements track the lower branch of the multifrequency regime, right to the end of its existence. This is a consequence of the experimental protocol in which $H$ is fixed. Presumably the other frequency branches could be accessed by varying both $H$ and $U$, or perhaps by making finite perturbations to the state of the thread. The frequency branches have been observed in pure rope coiling experiments [5, 7. The lower branch and the observation of single frequency meandering both end near $H=7.0 \mathrm{~cm}$. Above this value, we find mostly multifrequency meandering at onset, as shown in Fig. 3 .

\section{Meandering amplitude saturation: a simple model}

The fits to the amplitude of meandering also give a quantity that is not predicted by linear theory, namely the coefficient $\mu$ of the cubic nonlinear term in Eqn. 4 . Fig. 8 shows the experimental result. We find that $\mu$ is a decreasing function of $H$. The coefficient $\mu$ controls the dependence of the saturated amplitude on the belt speed. It can be predicted by the following argument, which is 


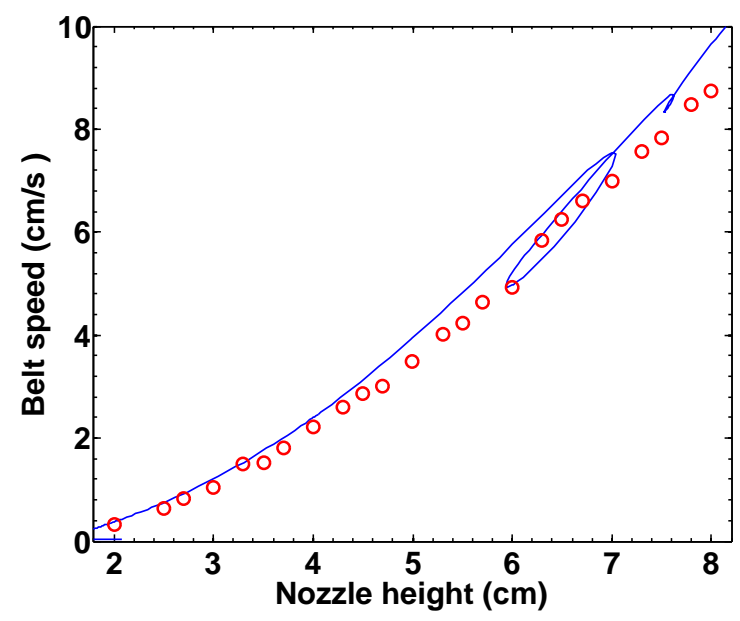

FIG. 5: (Color online) The critical belt speed $U_{c}$ vs $H$, extracted from fits to amplitudes like the one shown in the previous figure. The solid line is calculated from the linear stability analysis of Ref. [16], with no adjustable parameters.

based on symmetry and the assumption of a constant contact-point speed.

We employ coordinates in the lab frame with $x$ longitudinal to the belt, $y$ transverse to it and $z$ up. Ribe et al. [16] observed that the linear perturbation equations about a steady stretched catenary decouple into disjoint systems for perturbations in the plane $(x, z)$ of the catenary and those out of the $(x, z)$-plane. Meandering is an out-of-plane perturbation at linear order. We observe that the reflectional symmetry of the catenary further implies that the nonlinear perturbation equations are invariant to a change in the sign of all the out-of-plane variables. Hence if we perform a series expansion for the weakly nonlinear form of meandering near onset $(A \ll 1)$ then all the in-plane variables are even functions of $A$ and all the out-of-plane variables are odd functions of $A$. In particular, the contact point $(x(t), y(t))$ between thread and belt must have the form

$$
\begin{aligned}
& y(t)=A \sin (\omega t)+O\left(A^{3}\right), \\
& x(t)=x_{0}+B \cos (2 \omega t+\phi)+O\left(A^{4}\right),
\end{aligned}
$$

where $B=O\left(A^{2}\right), \omega=\omega_{c}+O\left(A^{2}\right), \phi$ is a constant phase, and $x_{0}$ is the unperturbed displacement plus an $O\left(A^{2}\right)$ correction. Thus, the absolute speed $V(t)$ of the contact point is given by

$$
\begin{aligned}
V^{2}= & (\dot{x}-U)^{2}+\dot{y}^{2}, \\
= & U^{2}+4 B \omega U \sin (2 \omega t+\phi) \\
& \quad+A^{2} \omega^{2}[1+\cos (2 \omega t)] / 2+O\left(A^{4}\right) .
\end{aligned}
$$

We now make the kinematic assumption that $V$ is constant and always equal to the critical belt speed $U_{c}$. This can be motivated either by the empirical observation in Ref. [16] that the steady speed of coiling is remarkably close to the belt speed at marginal stability, or by an

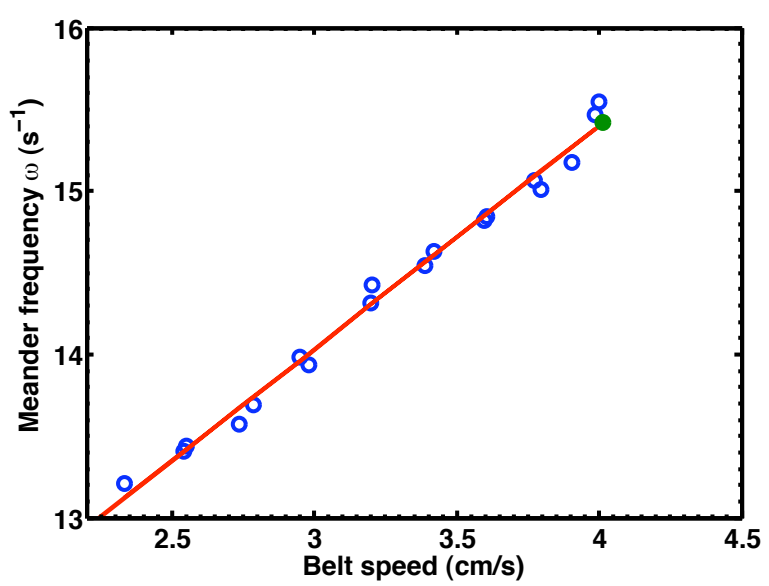

FIG. 6: (Color online) The angular frequency $\omega$ of the meandering motion (open circles), as a function of the belt speed $U$, for $H=5.3 \mathrm{~cm}$. The solid line is a fit to a linear variation of $\omega$ vs. $U$, consistent with a Hopf bifurcation. The solid circle marks the extrapolation to the critical value $\omega_{c}$.

asymptotic argument that the amount of stretching in the bending boundary layer of a slender thread is negligible [18]. Setting $V=U_{c}$ in Eqn. 6 gives

$$
\begin{gathered}
U_{c}^{2}=U^{2}+A^{2} \omega^{2} / 2+O\left(A^{4}\right), \\
B=A^{2} \omega /(8 U)+O\left(A^{4}\right), \quad \text { and } \quad \phi=\frac{\pi}{2}+O\left(A^{2}\right) .
\end{gathered}
$$

We rearrange Eqn. 7 ,

$$
A^{2}=2\left(U_{c}^{2}-U^{2}\right) / \omega^{2} \approx\left(4 U_{c}^{2} / \omega_{c}^{2}\right)\left[\left(U_{c}-U\right) / U_{c}\right],
$$

and compare with Eqn. 4 to predict

$$
\mu=\left(\omega_{c} / 2 U_{c}\right)^{2} .
$$

It is evident from Fig. 8 that this purely kinematic prediction of $\mu$ works remarkably well, particularly at larger heights. In effect, the buckling part of the thread behaves like an inextensible rope being played out onto the moving belt, though there is still extension in the falling part closer to the nozzle. The greater discrepancy between observation and kinematic prediction at lower heights is likely due to weaker scale separation between the falling and buckling parts of the thread.

It would be interesting to examine the motion of an actual inextensible, flexible rope falling on the moving belt. Recently, the classic liquid rope-coiling instability has been compared to a real rope in just this way [19. Presumably, the kinematic condition on the rope at the point of contact with the surface of the belt would have to be enforced by their frictional interaction.

The above kinematic assumption also makes predictions for the amplitude $B$ and phase $\phi$ of a small, frequency $2 \omega$, longitudinal oscillation. The existence of this oscillation can be discerned in the spacetime plot of meandering viewed from the side, shown in Fig. 9(a), which 


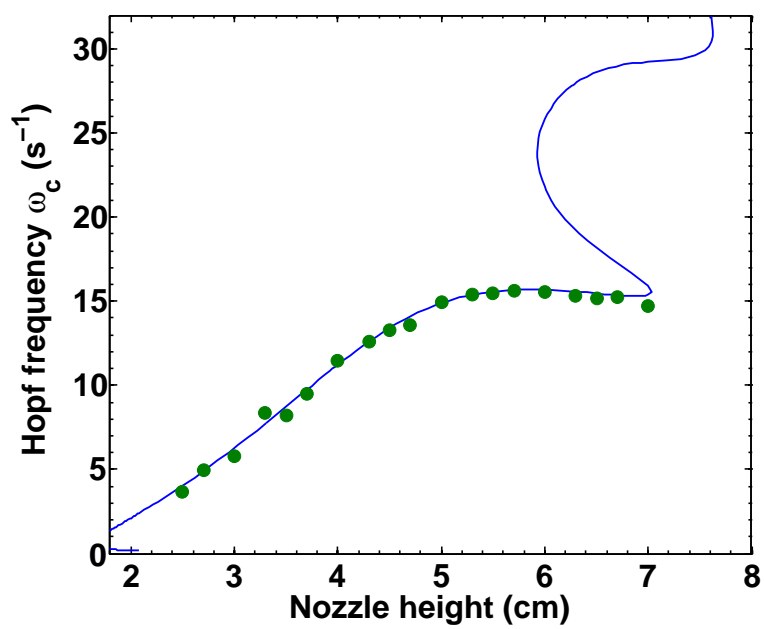

FIG. 7: (Color online) Measurements of the Hopf frequency $\omega_{c}$ (solid circles) as a function of the nozzle height $H$. The solid line is the theoretical result from the linear stability calculation of Ref. [16]. The reversals in the theoretical curve mark the multifrequency regime.

is discussed in more detail below. The experimental resolution of $B$ is however insufficient to make a quantitative comparison to theory.

The form of Eqn. 5 is justified from consideration of the full amplitude equations, which are presented in Section III.

\section{Beyond meandering: the figure eight and translated coiling states}

For $H \leq 5.5 \mathrm{~cm}$, the meandering state gives way at a non-hysteretic bifurcation to the figure eight state shown in Fig. 2. The figure eight state is, in its turn, unstable to the translated coiling state at lower $H$. This state links directly to pure fluid rope coiling at $H=0$. It is difficult to extract quantitative information about these states from photos of the "stitch pattern" on the belt. However, by viewing the thread from the side, the $x(t)$ and $y(t)$ components of motion can be measured. We accomplished this by positioning a small mirror at $45^{\circ}$ to the belt, so that a camera could image both the longitudinal and the transverse components at a point close to the location of maximum curvature of the thread, which was a few $\mathrm{mm}$ above the surface of the belt. Taking a single horizontal video line from such an image and plotting it in time gives spacetime images like those in Fig. 9

In these images, it is apparent that the meandering state consists of essentially a single frequency $\omega$ in $y$, with a small component at $2 \omega$ in $x$ with amplitude $B \ll A$, as assumed in Eqn. 5 above. The bifurcation to the figure eight state clearly involves the excitation of a large $2 \omega$ mode in $y$, which mixes with the $\omega$ mode in $x$ to produce the figure eight pattern. At the bifurcation to translated

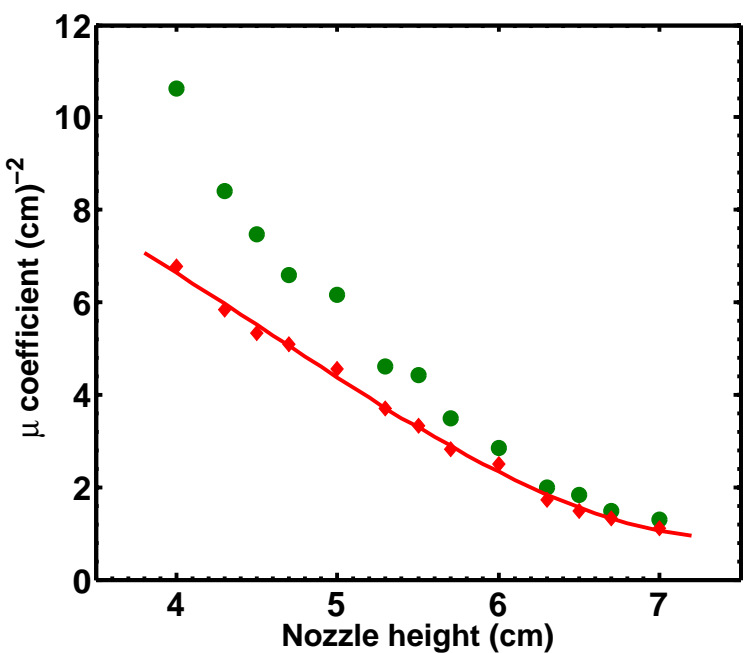

FIG. 8: (Color online) The nonlinear saturation coefficient $\mu$, as a function of the nozzle height $H$. The solid circles are the values deduced from fits to Eqn. 4. Diamond symbols show the kinematic estimate $\mu \approx\left(\omega_{c} / 2 U_{c}\right)^{2}$ for each height, discussed in the text. The solid line is a cubic polynomial model of the kinematic estimates.

coiling at lower $H$, the $2 \omega$ mode amplitude becomes small again, and the state is due to $x$ and $y$ oscillations at $\omega$ which are nearly $90^{\circ}$ out of phase.

These qualitative observations suggest that the meandering - figure eight - translated coiling series of transitions, and by extension all of the states of motion of the thread, could be understood in the framework of complex amplitude equations for modes with a few frequencies and their harmonics. These frequencies are probably closely related to the frequencies of pure coiling, including those in the multifrequency regime.

\section{AMPLITUDE EQUATIONS AND SYMMETRY}

We now discuss a more abstract approach to understanding the "stitch patterns" through the machinery of equivariant bifurcation theory 20. This theory offers a formal way to derive appropriate amplitude equations, starting from symmetry considerations alone. Such a qualitative approach offers a complementary perspective on the dynamics to that gained from fluid mechanical treatments. It also provides insight into the overall organisation of the bifurcations between these highly symmetric states. Given the rich phenomenology of this system, it seems wise to follow this route rather than attempt to account for all the states de novo from fluid mechanics alone. Although a rather large number of amplitudes may be required, this approach is still much simpler than the alternative. In principle, the full fluid mechanical description could be systematically projected 
onto a few modes, and the coefficients of the amplitude equations calculated. Alternatively, the coefficients could be deduced directly from experimental data of the kind shown in Fig. 9 .

We conjecture that the dynamics of the thread is close to the axisymmetric case of pure rope coiling, so that the motion of the belt can be treated as a perturbation. The moving belt breaks the rotational symmetry while preserving a reflection symmetry in a vertical plane containing the direction of travel of the belt. In bifurcationtheoretic terms, the onset of meandering from the steady catenary state is a Hopf bifurcation with weakly-broken $O(2)$ symmetry [21]. We denote the frequency at the Hopf bifurcation by $\omega$. To describe the secondary bifurcation in which the figure eight states appear, a minimal description must include amplitude equations for a mode with frequency $2 \omega$. We argue in the Appendix that the physical symmetries provide enough constraints to determine the form of the relevant amplitude equations. In section IIIA we discuss the effect of a further symmetry property which, while certainly not an exact symmetry of the physical problem, appears to help explain certain intriguing aspects of the dynamics of the viscous thread.

We propose an abstracted description of the fluid mechanical problem in which we focus on the position of the contact point of the viscous thread on the belt. We express the contact position of the viscous thread in the lab frame $(x(t), y(t))$ as the complex position coordinate

$$
\begin{aligned}
p(t)= & x(t)+i y(t) \\
= & A_{+}(t) \mathrm{e}^{i \omega t}+A_{-}(t) \mathrm{e}^{-i \omega t} \\
& +B_{+}(t) \mathrm{e}^{2 i \omega t}+B_{-}(t) \mathrm{e}^{-2 i \omega t},
\end{aligned}
$$

where $A_{+}, A_{-}, B_{+}$and $B_{-}$are the complex amplitudes of four rotating wave modes. By symmetry, the generic amplitude equations describing the dynamics, truncated at cubic order, take the form

$$
\begin{aligned}
\dot{A}_{+}= & A_{+}\left(\epsilon_{1}+i \omega_{1}+a_{1}\left|A_{+}\right|^{2}+a_{2}\left|A_{-}\right|^{2}\right. \\
& \left.+b_{1}\left|B_{+}\right|^{2}+b_{2}\left|B_{-}\right|^{2}\right)+s_{1} \bar{A}_{-} B_{+} B_{-} \\
& +\epsilon_{b}\left(e_{1} \bar{A}_{-}+p_{1} \bar{A}_{+} B_{+}\right. \\
& \left.+q_{1} \bar{A}_{+} \bar{B}_{-}+p_{3} A_{-} B_{+}+q_{3} A_{-} \bar{B}_{-}\right) \\
\dot{B}_{+}= & B_{+}\left(\epsilon_{2}+i \omega_{2}+c_{1}\left|B_{+}\right|^{2}+c_{2}\left|B_{-}\right|^{2}\right. \\
& \left.+d_{1}\left|A_{+}\right|^{2}+d_{2}\left|A_{-}\right|^{2}\right)+s_{2} \bar{B}_{-} A_{+} A_{-} \\
& +\epsilon_{b}\left(e_{2} \bar{B}_{-}+p_{2} \bar{A}_{-}^{2}+q_{2} A_{+}^{2}+p_{4} A_{+} \bar{A}_{-}\right)
\end{aligned}
$$

and two similar equations for $A_{-}$and $B_{-}$, given in the Appendix. The coefficients of the nonlinear terms in Eqns. 12 and 13 are in general complex-valued, and overbars denote complex conjugates. The bifurcation parameters $\epsilon_{1}$ and $\epsilon_{2}$ are complicated unknown functions of the dimensionless groups identified above, or equivalently of the natural experimental control parameters $U$ and $H$. $\epsilon_{1}\left(U_{c}, H\right)=0$ at the onset of meandering, as described
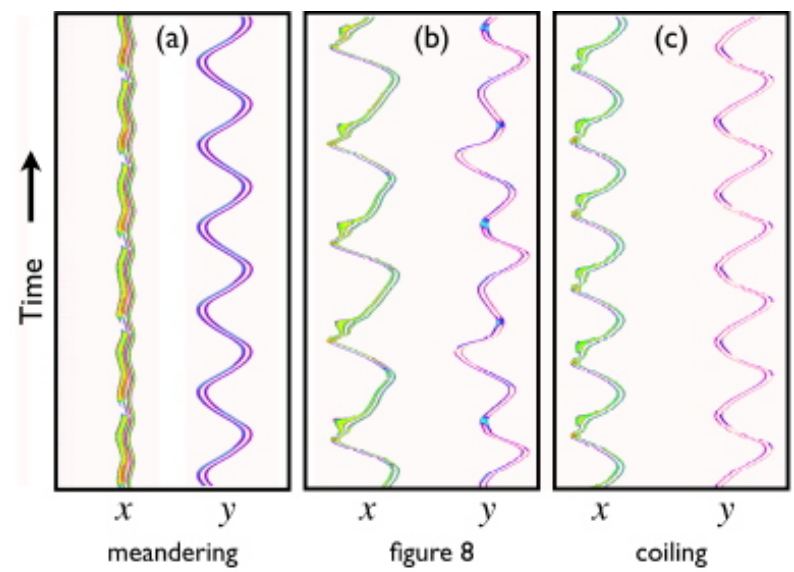

FIG. 9: (Color online) Spacetime views of the side of the thread, with time running upward. $x$ is a coordinate longitudinal to the belt, while $y$ is transverse. (a) shows the meandering state, where almost all the motion is transverse. (b) shows the figure eight state in which two harmonics of the basic frequency are clearly visible in the longitudinal motion. (c) shows translated coiling, in which one main harmonic appears, with a phase difference between $x$ and $y$.

above in section IIB $\epsilon_{2}\left(U_{c}, H\right)$ remains negative and describes the linear damping of the $2 \omega$ mode. The parameter $\epsilon_{b}$, a function of $U$ with $\epsilon_{b}(U=0)=0$, controls the strength of the symmetry-breaking effects of the belt on the thread.

Eqns. 12 and 13 justify the use of the phenomenological Eqns. 4 and 5, as we discuss further below. A full analysis of the bifurcation structure of Eqns. 12 and 13 is mathematically feasible, but beyond the scope of this paper. In the remainder of this section, we first discuss the meandering instability, and then the transition from meandering to figure eights, in the context of these amplitude equations.

The steady catenary solution $A_{+}=A_{-}=B_{+}=$ $B_{-}=0$ exists for all parameter values and is stable if $\epsilon_{1} \pm \epsilon_{b} \operatorname{Re}\left(e_{1}\right)<0$. Experimentally, there is a critical height $H_{0} \approx 2.0 \mathrm{~cm}$ below which no rope coiling instability occurs for $U=0$. It is therefore natural to speculate that, for small $U$ and heights close to $H_{0}$, we could assume $\epsilon_{1} \propto\left(H-H_{0}\right)$ and $\epsilon_{b} \propto U$. These scalings in turn predict a straight-line marginal stability boundary for the onset of meandering near this endpoint. This regime is difficult to reach experimentally, however, due to the extremely slow time evolution and the thickening of the thread relative to its meandering amplitude.

In the axisymmetric case $U=0$, when $\epsilon_{b}=0$, there is an $O(2)$-symmetric Hopf bifurcation at $H=H_{0}$ in which two oscillatory branches appear [22]: a rotating wave corresponding to coiling, and a standing wave corresponding to meandering. The coiling state persists under weak symmetry-breaking [21] and evolves smoothly into the translated coiling state $A_{+} \neq 0, A_{-}=B_{+}=B_{-}=0$, observed for small $U$. In the axisymmetric case, the bi- 
furcating standing wave has no preferred horizontal orientation. Under weak symmetry-breaking, two standing waves out of this family of states survive. One of these states corresponds to oscillations where the thread lies entirely within the symmetry-plane of the belt at all times (a "longitudinal oscillation"), the other ("meandering") has the combination spatiotemporal symmetry $\kappa \circ \tau_{\pi}$ (defined precisely in the Appendix) of reflection across the belt followed by waiting for a half-period of the oscillation.

The figure eight state also has the symmetry $\kappa \circ \tau_{\pi}$ and so, to simplify the further discussion of meandering and figure eights, we restrict our attention only to solutions that have the $\kappa \circ \tau_{\pi}$ symmetry, that is, which have the form $A_{+}=-\bar{A}_{-} \equiv A$ and $B_{+}=\bar{B}_{-} \equiv B$. We refer to the invariant subspace $(A,-\bar{A}, B, \bar{B})$ as $\mathcal{S}$; importantly, $\mathcal{S}$ remains invariant even if higher-order terms are added to the amplitude equations.

Within $\mathcal{S}$, the amplitude equations simplify to become

$\dot{A}=\left(\epsilon_{1}+i \omega_{1}-\epsilon_{b} e_{1}\right) A+i \epsilon_{b} \alpha_{1} \bar{A} B+\beta_{1} A|A|^{2}+\beta_{2} A|B|^{2}$,

$\dot{B}=\left(\epsilon_{2}+i \omega_{2}+\epsilon_{b} e_{2}\right) B+i \epsilon_{b} \alpha_{2} A^{2}+\beta_{3} B|B|^{2}+\beta_{4} B|A|^{2}$.

The coefficients $e_{1}, e_{2}, \alpha_{1}, \alpha_{2}, \beta_{1}, \ldots, \beta_{4}$ in Eqns. 14 and 15 can be deduced by direct comparison with Eqns. 12 and 13. Near the initial meandering instability $\epsilon_{1}-\epsilon_{b} \operatorname{Re}\left(e_{1}\right)=0$, and we may eliminate $B$ adiabatically, or via a centre manifold reduction, so that to leading order $B=-i \epsilon_{b} \alpha_{2} A^{2} /\left(\epsilon_{2}+i \omega_{2}+\epsilon_{b} e_{2}\right)+O\left(A^{3}\right)$ and hence

$\dot{A}=\left(\epsilon_{1}+i \omega_{1}-\epsilon_{b} e_{1}\right) A+\left(\frac{\epsilon_{b}^{2} \alpha_{1} \alpha_{2}}{\epsilon_{2}+i \omega_{2}+\epsilon_{b} e_{2}}+\beta_{1}\right) A|A|^{2}$

(up to $O\left(A^{3}\right)$ ), which is the usual amplitude equation for a Hopf bifurcation, justifying the use of Eqn. 4

Writing $A=R_{1} \mathrm{e}^{i \theta_{1}}$ and $B=R_{2} \mathrm{e}^{i \theta_{2}}$ we find that, due to the time-translation symmetry, Eqns. 14 and 15 reduce further, to a three-dimensional set of ODEs for $R_{1}, R_{2}$ and the relative phase $\chi=\theta_{2}-2 \theta_{1}$. There are regions of parameter space in which time-periodic solutions with a constant relative phase $\chi$ do not exist. In other regions of parameter space, there will typically be two possible constant values $\chi=\chi_{ \pm}$. In general, within $\mathcal{S}$ we recover the form

$$
\begin{aligned}
& y(t)=2 R_{1} \sin \omega t, \\
& x(t)=2 R_{2} \cos (2 \omega t+\chi),
\end{aligned}
$$

for the location of the tip of the thread, which confirms Eqn. 5 .

If $\epsilon_{1}>0$ and $\epsilon_{2}<0$ (as expected for the onset of meandering when $H<6.0 \mathrm{~cm}$ ), and if $\epsilon_{b}=0$, we would expect stable meandering to exist for $0<\epsilon_{1}<$ $\operatorname{Re}\left(\beta_{1}\right) \epsilon_{2} / \operatorname{Re}\left(\beta_{4}\right)$, choosing $\operatorname{Re}\left(\beta_{1}\right)<0$ to ensure that the onset of meandering is supercritical, and $\operatorname{Re}\left(\beta_{4}\right)>0$ to ensure the existence of an instability of meandering to the $2 \omega$ mode that produces the figure eight pattern. At $\epsilon_{1}=\operatorname{Re}\left(\beta_{1}\right) \epsilon_{2} / \operatorname{Re}\left(\beta_{4}\right)$ there is a pitchfork bifurcation at which meandering loses stability to "mixed-modes" involving the $2 \omega$ mode. For $\epsilon_{b} \neq 0$ the pitchfork bifurcation becomes imperfect, with a continuous, but rapid and non-hysteretic, increase in the amplitude of the $2 \omega$ mode in the vicinity of $\epsilon_{1}=\operatorname{Re}\left(\beta_{1}\right) \epsilon_{2} / \operatorname{Re}\left(\beta_{4}\right)$.

Thus, the sequence of transitions observed experimentally has a natural interpretation in terms of the mode interaction described above.

Moreover, the experimental evidence indicates a discontinuous but not hysteretic transition from meandering to figure eight; from the theory one would expect either that the transition is indeed abrupt (for example a subcritical bifurcation) and accompanied by measurable hysteresis, or that the transition is non-hysteretic but continuous. The resolution of the exact nature of the transition from meandering to the figure eight state will require better controlled experiments to sort out. Direct measurement of the amplitudes and phases of the $\omega$ and $2 \omega$ components of the pattern should enable this intriguing discrepancy to be resolved.

\section{A. An additional model symmetry}

The amplitude equations, discussed above, do not precisely determine the relative phase $\chi$. However, experimental results (Fig. 2 as well as those of Ref. [15]) indicate that the values $\chi=\pi / 2$ ( the "bunched-up meandering" state discussed in Ref. [15) and near $\chi=-\pi / 2$ (figure eight) are consistently selected. It is natural to ask whether there is a reason for this, either of an abstract or a fluid-mechanical nature.

As far as the abstract model problem goes, it turns out that there is indeed an additional symmetry that implies $\chi= \pm \pi / 2$. This symmetry (labelled $h$ in the Appendix) is a $180^{\circ}$ rotation of the $(x, y)$ plane followed by reversal of the direction of time. Clearly this is not a symmetry of the original physical problem: in no sense are we suggesting that falling fluid in the thread is related to a viscous thread of rising fluid! But in the bifurcation-theoretic model, we ignore the vertical direction, and the timereversal applied to the equilibrium state results only in the reversal of the direction of travel of the belt. Even so, we would expect $h$ to be only an approximate symmetry since the initial steady catenary state is not symmetric under a $180^{\circ}$ rotation. The consequences of the extra symmetry $h$ would be to force the imaginary parts of all coefficients in Eqns. 14 and 15 to be real. In this case, the ODEs for $R_{1}, R_{2}$ and the relative phase $\chi=\theta_{2}-2 \theta_{1}$ 
become

$$
\begin{aligned}
& \dot{R}_{1}=\left(\epsilon_{1}-\epsilon_{b} e_{1}\right) R_{1}-\epsilon_{b} \alpha_{1} R_{1} R_{2} \sin \chi+\beta_{1} R_{1}^{3}+\beta_{2} R_{1} R_{2}^{2}, \\
& \dot{R}_{2}=\left(\epsilon_{2}+\epsilon_{b} e_{2}\right) R_{2}+\epsilon_{b} \alpha_{2} R_{1}^{2} \sin \chi+\beta_{3} R_{2}^{3}+\beta_{4} R_{2} R_{1}^{2}, \\
& \dot{\chi}=\epsilon_{b}\left(\frac{\alpha_{2} R_{1}^{2}}{R_{2}}-2 \alpha_{1} R_{2}\right) \cos \chi .
\end{aligned}
$$

Hence, from Eqn. 20, time-periodic solutions with a constant relative phase exist only for $\chi= \pm \pi / 2$. If the coefficients in Eqns. 14 and 15 have (small) nonzero imaginary parts then the two time-periodic states persist but now only have $\chi \approx \pm \pi / 2$. There is some experimental evidence that the bunched-up meandering state observed in Ref. [15] has $\chi \approx \pi / 2$, without having exact equality.

More generally, Eqns. 18 - 20 are equivalent to the amplitude equations of the well-studied $1: 2$ steady-state mode interaction [23, 24, 25, 26]. The meandering and figure eight states correspond to "mixed-mode" equilibria in this language, since both states contain non-zero amounts of both the $\omega$ and $2 \omega$ modes. However, the analysis in Refs. 23, 24, 25, 26] concentrates on cases where the quadratic terms have order unity coefficients and strongly influence the dynamics near $R_{1}=R_{2}=0$. In contrast, in the present case, we are interested in the regime $\epsilon_{b} \ll 1$ where we see from Eqns. 18 - 20 that the quadratic terms fix the relative phase $\chi$ but only weakly affect the amplitudes $R_{1}$ and $R_{2}$.

Having derived the form of the amplitude equations on symmetry grounds, an obvious next step would be to compute or measure the undetermined coefficients in them. It is straightforward to extend Eqns. 12 and 13 to account for higher frequencies, although the number of terms, even at cubic order, grows quite rapidly. Projecting the full dynamics of the thread onto these equations serves to simplify the problem to finding the coefficients and how they depend on the nondimensional experimental parameters $H\left(g / \nu^{2}\right)^{1 / 3}, U\left(g H^{2} / \nu\right)$ and $\Pi_{1}, \Pi_{2}$ and $\Pi_{3}$ given by Eqns. 1 13 . In principle, the entire state diagram shown in Fig. 3 may be understood in this way.

\section{CONCLUSIONS}

In this paper we have reported a detailed experimental survey of the "stitch patterns" observed in a more precise version of the "fluid-mechanical sewing machine" [15. We studied, in particular, the onset of the meandering state and measured its critical belt speed, Hopf frequency and amplitude saturation. Our theoretical understanding and interpretation of this instability of the catenary thread comes from three sources: a detailed numerical solution of the linear stability problem [16, an ad hoc argument which used the assumption that the contact point of the thread with the belt has a constant velocity, and the construction of the full amplitude equations on symmetry grounds.
We found that the onset of meandering is very well described as a Hopf bifurcation with the critical parameters $U_{c}$ and $\omega_{c}$ accurately predicted by a linear stability analysis of the fluid equations [16. Surprisingly, the nonlinear saturation of the meandering motion could be mostly accounted for by simply assuming that the fluid thread behaves as an inextensible rope being played out onto the belt. This is the case despite the considerable buckling and stretching of the real thread. Finally, the full amplitude equations, derived from symmetry considerations independent of the fluid mechanical details, provide a systematic way of organizing and interpreting the otherwise rather complex motion of the thread.

From the full amplitude equations we were able to explain, at least in outline, the existence of the figure eight bubble that occurs over a substantial part of the state diagram for smaller $H$, as seen in Fig. 3. It seems likely that this state is a manifestation of a $1: 2$ steadystate mode interaction [23, 24, 25, 26]. In general, such a mode interaction is well known to generate complex dynamics in certain cases, such as when $\alpha_{1}$ and $\alpha_{2}$ in Eqns. 18 - 20 have opposite signs. In this case, one expects quasiperiodic oscillations and robust heteroclinic cycling. Physically, such dynamical complexity would be most easily observed in the intermittent evolution of the relative phase variable $\chi$ on a timescale that is slow compared to the basic meandering oscillation period. Future, improved experiments could look for such subtle effects.

An interesting question that needs to be addressed more fully, both in experiment and theory, is the variation of the oscillation frequency as the belt speed is reduced. Ribe et. al. [16] compared the frequency at the onset of meandering to the frequency of finite amplitude "pure" coiling (when $U=0$ ) at the same height $H$ and found excellent agreement. Our results, however, indicate that the meandering frequency decreases substantially from that at onset as the belt speed decreases, as shown in Fig. 6. This decrease is exactly what is expected from the linear amplitude dependence of the frequency following a Hopf bifurcation. But how can this be reconciled with the requirement that the frequency must also agree with the pure coiling frequency again when $U$ is ultimately reduced to zero? Is it the case that the frequency reaches a local minimum as $U$ is decreased, before increasing continuously back towards pure coiling, or are there discontinuities? What is the effect of the intervening figure eight state on the fundamental frequency?

For larger values of $H$, the amplitude equation model would have to be extended to allow for modes with the additional frequencies that arise from the new pendulum motions of the thread. Using data gathered from sideview images like that shown in Fig. 9, amplitudes and phases of the constituent motions of the thread could be acquired and interpreted within the framework of the predictions of the amplitude equations. Future work using this approach will require a more complete investigation of the bifurcation structure of multifrequency amplitude equations with weakly broken $O(2)$ symmetry. 


\section{Acknowledgments}

This work was supported by the George Batchelor Laboratory, University of Cambridge. We acknowledge useful discussions with Sunny Chiu-Webster, Sue Colwell, and Stuart Dalziel. We are grateful to Lucas Goehring for the rheometry and densitometry of the silicon oil. JHPD is supported by Newnham College, Cambridge and the Royal Society.

\section{APPENDIX: RESONANT HOPF BIFURCATION WITH WEAKLY BROKEN $O(2)$ SYMMETRY}

In this Appendix, we briefly sketch the derivation of the abstract amplitude equations, Eqns. 12 and 13 , describing the dynamics for heights $H$ towards the lower end of the range investigated experimentally. We emphasize that this discussion proceeds in a manner independent of the fluid-mechanical details of the flow, and, although it provides some support for our experimental results and simple explanatory ansatz, it remains a modelling approach.

The system is taken to be a weak perturbation of the axisymmetric case that corresponds to $U=0$. We first compute the amplitude equations for the axisymmetric case and then discuss how the weak symmetry-breaking provided by the belt admits additional coupling terms.

In the axisymmetric case, the symmetry group consists of planar rotations about the origin, and reflections in vertical planes containing the origin: this is the orthogonal group $O(2)$. The action of $O(2)$ on the plane $\mathbb{R}^{2}$ containing the belt is generated by the elements $\kappa$ (a reflection in the plane containing the belt) and $\rho_{\theta}$ (rotation through angle $\theta$ ). Identifying $(x, y) \in \mathbb{R}^{2}$ with $p=x+i y \in \mathbb{C}$ we have

$$
\kappa(p)=\bar{p}, \quad \rho_{\theta}(p)=\mathrm{e}^{i \theta} p .
$$

In addition, the system has no preferred origin in time, and hence the time-periodic solutions we investigate have no preferred phase. This additional symmetry $\tau_{\phi}: t \rightarrow$ $t+\phi / \omega$ generates a group $S^{1}$ corresponding to phase shifts of the amplitudes. We suppose that the coiling motion can be described as a sum of four oscillatory amplitudes for a complex position coordinate, as in Eqn. 11 . Let $\mathbf{A}=\left(A_{+}, A_{-}, B_{+}, B_{-}\right)$denote the vector of amplitudes. The action of the full symmetry group $O(2) \times S^{1}$ on $\mathbf{A}$ is inherited from the action on $\mathbb{R}^{2}$ of the generators $\left(\kappa, \rho_{\theta}\right)$, and the phase-shift symmetry $\tau_{\phi}$ :

$$
\kappa\left(A_{+}, A_{-}, B_{+}, B_{-}\right)=\left(\bar{A}_{-}, \bar{A}_{+}, \bar{B}_{-}, \bar{B}_{+}\right),
$$

$\rho_{\theta}\left(A_{+}, A_{-}, B_{+}, B_{-}\right)=\mathrm{e}^{i \theta}\left(A_{+}, A_{-}, B_{+}, B_{-}\right)$,

$\tau_{\phi}\left(A_{+}, A_{-}, B_{+}, B_{-}\right)=\left(\mathrm{e}^{i \phi} A_{+}, \mathrm{e}^{-i \phi} A_{-}, \mathrm{e}^{2 i \phi} B_{+}, \mathrm{e}^{-2 i \phi} B_{-}\right)$.

The required amplitude equations

$$
\dot{\mathbf{A}}=\mathbf{F}(\mathbf{A}),
$$

that are symmetric under Eqns. A.1-A.3 obey the equivariance condition $\gamma \mathbf{F}(\mathbf{A})=\mathbf{F}(\gamma \mathbf{A})$, for all group elements $\gamma \in O(2) \times S^{1}$. The equivariance condition is a mathematical statement of the intuitive notion that whenever $\mathbf{A}(t)$ is a solution of the amplitude equations, we require $\gamma \mathbf{A}(t)$ also to be a solution trajectory. We can now determine the form of the most general amplitude equations by computing invariant and equivariant polynomials. General methods for this exist [20] but are too cumbersome to apply here, particularly since we intend only to compute terms at the first few orders. As usual in problems of this type, the terms $\left|A_{+}\right|^{2},\left|A_{-}\right|^{2},\left|B_{+}\right|^{2}$ and $\left|B_{-}\right|^{2}$ (and sums and products of these) are invariant. To find other invariants we adopt the "brute force" approach of looking for an invariant term $A_{+}^{a} A_{-}^{b} B_{+}^{c} B_{-}^{d}$ with integer powers, using the convention that a negative power denotes a positive power of the complex conjugate variable. Invariance under $\rho_{\theta}$ and $\tau_{\phi}$ implies $a+b+c+d=0$ and $a-b+2(c-d)=0$. We define the order of the invariant term to be $|a|+|b|+|c|+|d|$. The invariants of order 2 are $\left|A_{+}\right|^{2},\left|A_{-}\right|^{2},\left|B_{+}\right|^{2}$ and $\left|B_{-}\right|^{2}$. It is easily seen that the only invariant of order 4 that is not a product of order 2 invariants is $\bar{A}_{+} \bar{A}_{-} B_{+} B_{-}$. It follows that the terms up to cubic order in the first component $\dot{A}_{1}=F_{1}\left(A_{+}, A_{-}, B_{+}, B_{-}\right)$of A.4 will be

$$
\begin{array}{lll}
A_{+}, & A_{+}\left|A_{+}\right|^{2}, & A_{+}\left|A_{-}\right|^{2}, \\
A_{+}\left|B_{+}\right|^{2}, & A_{+}\left|B_{-}\right|^{2}, & \bar{A}_{-} B_{+} B_{-} .
\end{array}
$$

By now applying the reflection symmetry $\kappa$, we may deduce the second amplitude equation $F_{2}\left(A_{+}, A_{-}, B_{+}, B_{-}\right)$. Note that $\kappa$ implies that the coefficients in $F_{2}$ are the complex conjugates of those in $F_{1}$.

Similarly we deduce the order 2 and order 4 invariants for $F_{3}$ and then use $\kappa$ to compute $F_{4}$ from $F_{3}$. The resulting amplitude equations, Eqns. 12 and 13 with $\epsilon_{b} \equiv 0$, describe the axisymmetric interaction of the $\omega$ and $2 \omega$ modes.

The introduction of a (slowly-moving) horizontal belt changes the symmetry of the problem in two ways. The first of these is clear: it breaks the rotation symmetry $\rho_{\theta}$. To account for this effect we compute low-order terms that are not symmetric under $\rho_{\theta}$ but which do respect the remaining symmetry $\tau_{\phi}$. The eight new invariants are:

$$
\begin{aligned}
& A_{+} A_{-}, \quad B_{+} B_{-}, \quad \bar{A}_{+} A_{-} B_{+}, \quad A_{-}^{2} B_{+}, \\
& A_{+}^{2} \bar{B}_{+}, \quad A_{+} \bar{A}_{-} B_{-}, \quad A_{-}^{2} \bar{B}_{-}, \quad A_{+}^{2} B_{-} .
\end{aligned}
$$

The new quadratic invariants give rise to the new linear terms in Eqns. 12 and 13 and so split the symmetric Hopf bifurcation at $\epsilon_{1}=0$ into two generic Hopf bifurcations at $\epsilon_{1} \pm \epsilon_{b} \operatorname{Re}\left(e_{1}\right)=0$. The new cubic invariants generate the new quadratic terms. The amplitude equations for the four amplitudes are then given by Eqn. 12 and 13 
along with

$$
\begin{aligned}
\dot{A}_{-}= & A_{-}\left(\epsilon_{1}-i \omega_{1}+\bar{a}_{1}\left|A_{-}\right|^{2}+\bar{a}_{2}\left|A_{+}\right|^{2}\right. \\
& \left.+\bar{b}_{1}\left|B_{-}\right|^{2}+\bar{b}_{2}\left|B_{+}\right|^{2}\right)+\bar{s}_{1} \bar{A}_{+} B_{+} B_{-} \\
& +\epsilon_{b}\left(\bar{e}_{1} \bar{A}_{+}+\bar{p}_{1} \bar{A}_{-} B_{-}\right. \\
& \left.+\bar{q}_{1} \bar{A}_{-} \bar{B}_{+}+\bar{p}_{3} B_{-} A_{+}+\bar{q}_{3} A_{+} \bar{B}_{+}\right), \\
\dot{B}_{-}= & B_{-}\left(\epsilon_{2}-i \omega_{2}+\bar{c}_{1}\left|B_{-}\right|^{2}+\bar{c}_{2}\left|B_{+}\right|^{2}\right. \\
& \left.+\bar{d}_{1}\left|A_{-}\right|^{2}+\bar{d}_{2}\left|A_{+}\right|^{2}\right)+\bar{s}_{2} \bar{B}_{+} A_{+} A_{-} \\
& +\epsilon_{b}\left(\bar{e}_{2} \bar{B}_{+}+\bar{p}_{2} \bar{A}_{+}^{2}+\bar{q}_{2} A_{-}^{2}+\bar{p}_{4} \bar{A}_{+} A_{-}\right)(A
\end{aligned}
$$

Some features of the phase relationship between the $\omega$ and $2 \omega$ modes suggest the introduction of an additional symmetry, denoted $h$ : rotating the apparatus by $180^{\circ}$ around the vertical $z$-axis and reversing the direction of travel of the belt (i.e. reversing the direction of time) leaves the basic state $p=0$ unchanged. For the bifurcation problem constructed in this Appendix, $h$ is an independent symmetry that introduces a new constraint. But $h$ is clearly not an exact symmetry of the original fluidmechanical problem where the fluid in the viscous thread falls under gravity, and the initial equilibrium state is a catenary rather than an exactly vertical thread. So, at best, the effect of $h$ is felt in some approximate way by amplitude equations describing the original physical system.
The new symmetry $h(x, y, t)=-(x, y, t)$ acts on the mode amplitudes as

$$
h\left(A_{+}, A_{-}, B_{+} \cdot B_{-}\right)=-\left(A_{-}, A_{+}, B_{-}, B_{+}\right)
$$

So the composite operation $h \circ \kappa$ sends each amplitude to the negative of its complex conjugate; equivariance of Eqn. A.4 under $h \circ \kappa$ implies that odd order terms would have real coefficients and even order terms would have purely imaginary coefficients. More realistically, we might hope that the coefficients have non-zero but small (compared to their modulus) imaginary or real parts at odd and even orders respectively.

The analysis of Eqns. 12, 13, A.5 and A.6 is simplified by the existence of invariant subspaces for the dynamics technically, the fixed-point subspaces of group elements. The most relevant of these for this paper consists of those points left unchanged by the combined operation $\kappa \circ \tau_{\pi}$ (reflection in the plane of the belt followed by a timeshift of half an oscillation period): it is therefore useful to define the invariant subspace

$$
\mathcal{S} \equiv \operatorname{Fix}\left(\kappa \circ \tau_{\pi}\right)=\left(A_{+},-\bar{A}_{+}, B_{+}, \bar{B}_{+}\right)
$$

which contains both the meandering and figure eight states.
[1] J. Ingram, The Velocity of Honey: And More Science of Everyday Life, (Penguin, London, 2003).

[2] G. I. Taylor, Proc. 12th Intl. Congr. Appl. Mech., Springer, Berlin, 382 (1969).

[3] G. Barnes and R. Woodcock, Am. J. Phys., 26, 205 (1958).

[4] G. Barnes and J. MacKenzie, Am. J. Phys., 27, 112 (1959).

[5] M. Maleki, M. Habibi, R. Golestanian, N. M. Ribe and D. Bonn, Phys. Rev. Lett., 93, 214502 (2004).

[6] J. O. Cruickshank and B. R. Munson, J. Fluid Mech., 113, 221 (1981).

[7] N. M. Ribe, H. E. Huppert, M. Hallworth, M. Habibi and D. Bonn, J. Fluid Mech. 555, 275 (2006).

[8] L. Mahadevan, W. S. Ryu and A. D. T. Samuel, Nature, 392, 140 (1998).

[9] L. Mahadevan, W. S. Ryu, and A. D. T. Samuel, Nature, 403, 502 (2000).

[10] J. O. Cruickshank, J. Fluid Mech., 193, 111 (1988).

[11] B. Tchavdarov, A. L. Yarin and S. Radev, J. Fluid Mech., 253, 593 (1993).

[12] M. Skorobogatiy and L. Mahadevan, Europhys. Lett., 52, $532(2000)$.

[13] N. M. Ribe, Proc. R. Soc. London, Ser. A 460, 3223 (2004).

[14] N. M. Ribe, M. Habibi and D. Bonn, Phys. Fluids 18,
084102 (2006).

[15] S. Chiu-Webster and J. R. Lister, J. Fluid Mech., 569, 89 (2006).

[16] N. M. Ribe, J. R. Lister and S. Chiu-Webster, Phys. Fluids 18, 124105 (2006).

[17] A. Hlod, A.C.T. Aarts, A. A. F. Van De Ven and M. A. Peletier, Eur. J. Appl. Math., 18, 659 (2007).

[18] M. J. Blount and J. R. Lister, personal communication.

[19] M. Habibi, N. M. Ribe and D. Bonn, Phys. Rev. Lett., 99, 154302 (2007)

[20] M. Golubitsky, I.N. Stewart and D.G. Schaeffer, Singularities and Groups in Bifurcation Theory. Volume II. Springer, Applied Mathematical Sciences Series 69 (1988)

[21] G. Dangelmayr and E. Knobloch, Nonlinearity, 4, 399 (1991)

[22] M. Golubitsky and I.N. Stewart, Arch. Rat. Mech. Anal. 87, 107 (1985)

[23] G. Dangelmayr, Dyn. Stab. Syst. 1, 159 (1996)

[24] D. Armbruster, J. Guckenheimer \& P. Holmes, Physica D 29, 257 (1987)

[25] M.R.E. Proctor and C.A. Jones, J. Fluid Mech. 188, 301 (1988)

[26] J.H.P. Dawes, C.M. Postlethwaite and M.R.E. Proctor, Physica D 191, 1 (2004) 